\title{
A novel phosphoglucomutase-deficient mouse model reveals aberrant glycosylation and early embryonic lethality
}

\author{
Bijina Balakrishnan $^{1}$ | Jan Verheijen ${ }^{2}$ | Arielle Lupo ${ }^{1}$ | Kimiyo Raymond ${ }^{2}$ | \\ Coleman Turgeon $^{2}$ | Yueqin Yang ${ }^{1}$ | Kandis L. Carter ${ }^{3}$ | Kevin J. Whitehead ${ }^{3}$ । \\ Tamas Kozicz $^{2}$ | Eva Morava ${ }^{2}$ । Kent Lai ${ }^{1}$ (๑)
}

${ }^{1}$ Department of Pediatrics, University of Utah School of Medicine, Salt Lake City, Utah

${ }^{2}$ Center for Individualized Medicine, Department of Clinical Genomics, and Biochemical Genetics Laboratory, Mayo Clinic, Rochester, Minnesota

${ }^{3}$ Small Animal Ultrasound Core Facility, University of Utah School of Medicine, Salt Lake City, Utah

\section{Correspondence}

Kent Lai, Division of Medical Genetics, Department of Pediatrics, University of Utah School of Medicine, 295 Chipeta Way, Salt Lake City, UT 84108.

Email: kent.lai@hsc.utah.edu

\section{Communicating Editor: Jaak Jaeken}

\section{Funding information}

National Center for Advancing Translational Sciences, Grant/Award Number: FP00096621; National Institutes of Health, Grant/Award Number: S10 RR027506-01; Primary Children's Hospital Foundation, Grant/Award Number: K2R2R; Undiagnosed Disease Network, Grant/ Award Number: Metabolomics Core

\begin{abstract}
Patients with phosphoglucomutase (PGM1) deficiency, a congenital disorder of glycosylation (CDG) suffer from multiple disease phenotypes. Midline cleft defects are present at birth. Overtime, additional clinical phenotypes, which include severe hypoglycemia, hepatopathy, growth retardation, hormonal deficiencies, hemostatic anomalies, frequently lethal, early-onset of dilated cardiomyopathy and myopathy emerge, reflecting the central roles of the enzyme in (glycogen) metabolism and glycosylation. To delineate the pathophysiology of the tissue-specific disease phenotypes, we constructed a constitutive Pgm2 (mouse ortholog of human PGM1)knockout (KO) mouse model using CRISPR-Cas9 technology. After multiple crosses between heterozygous parents, we were unable to identify homozygous life births in 78 newborn pups $(P=1.59897 \mathrm{E}-06)$, suggesting an embryonic lethality phenotype in the homozygotes. Ultrasound studies of the course of pregnancy confirmed Pgm2-deficient pups succumb before E9.5. Oral galactose supplementation ( $9 \mathrm{mg} / \mathrm{mL}$ drinking water) did not rescue the lethality. Biochemical studies of tissues and skin fibroblasts harvested from heterozygous animals confirmed reduced Pgm2 enzyme activity and abundance, but no change in glycogen content. However, glycomics analyses in serum revealed an abnormal glycosylation pattern in the $P g m 2^{+/-}$animals, similar to that seen in PGM1-CDG.
\end{abstract}

\section{K E Y W O R D S}

aberrant $N$-linked glycosylation, congenital disorders of glycosylation, embryonic lethality, galactose supplementation, inborn errors of metabolism, phosphoglucomutase 1 deficiency

\section{1 | INTRODUCTION}

Phosphoglucomutase 1 (PGM1) catalyzes the interconversion of glucose-1 phosphate (Glc-1P) and glucose-6 phosphate (Glc-6P) and therefore, it plays a fundamental role in glycolysis, glycogenesis, and glycogenolysis. ${ }^{1}$ Consequently, inherited deficiency of PGM1 in humans has previously been identified as glycogen storage disorder Type 14, which resulted from deleterious recessive variants in the $P G M 1$ genes. ${ }^{2,3}$ Yet, PGM1 deficiency is increasingly recognized as a congenital disorder of glycosylation (CDG), where reduced $\mathrm{N}$-linked glycosylation in the endoplasmic reticulum and Golgi is observed, resulting in both missing and truncated glycans (mixed type I and type II glycosylation defects). ${ }^{2,4-6}$

At birth, affected patients have frequent malformations in the spectrum of bifid uvula, cleft palate, and Pierre-Robin 
sequence. The liver, the skeletomuscular system, endocrine, and coagulation systems get all involved overtime, but the most life-threatening complication is the early-onset dilated cardiomyopathy, reflecting the central roles of the enzyme in glycogen metabolism and glycosylation ${ }^{7-10}$ (Figure 1). Recently, it has been shown that oral D-galactose supplementation in a growing cohort of patients with PGM1 deficiency improved serum transferrin hypoglycosylation, liver function, endocrine abnormalities, and reduced the frequency of hypoglycemic episodes. ${ }^{9,11-13}$ However, not all clinical abnormalities were corrected at the dosage used. A better understanding of the pathophysiology of PGM1 deficiency is needed to enable the development of more effective therapies for this disorder. As human PGM1 and mouse Pgm2 are $97.7 \%$ identical at the amino acid level and catalyze the same reactions, while the mouse Pgm1 converts ribose1-phosphate/deoxyribose-1-phosphate to the corresponding 5-phosphopentose, ${ }^{14}$ we examined the disruption of the Pgm2 gene in the mouse.

\section{2 | MATERIALS AND METHODS}

\section{1 | Construction of the constitutive Pgm2-KO mouse model}

Construction and characterization of the Pgm2-knockout $(K O)$ were conducted in accordance with the approved IACUC protocol.

Pgm2-KO mice were generated using the CRISPR/Cas9 approach at the Transgenic and Gene Targeting Mouse Core and the Mutation Generation and Detection Core, University of Utah. Guide RNAs (gRNAs) targeting exon 2 and exon 3 of $P g m 2$ were designed using CRISPOR (http://crispor. tefor.net, PMID: 27380939) and off-target sites were predicted using (http://casot.cbi.pku.edu.cn, PMID: 24389662). gRNA N(20) sequences used are as follows: CGAGGTCTACCTTCAAGTCA and GGATGATGCAAG ATACGGCA. The gRNAs were synthesized by making PCR templates that were transcribed with T7 RNA polymerase. $^{15}$

Super-ovulated C57B16/J embryos were harvested at E0.5, treated with hyaluronidase in M2 medium, and incubated in M16 medium under oil. CRISPR reagents were injected into the pronucleus of fertilized embryos. Two guide RNA's, PGM2-E2-S10 and PGM2-E3-S13, were diluted to $20 \mathrm{ng} / \mu \mathrm{L}$ each along with Cas 9 protein at $20 \mathrm{ng} /$ $\mu \mathrm{L}$ in RNase free water. Injected embryos were implanted into the oviduct of 0.5-day pseudo pregnant females and allowed to be born naturally. Pups were ear clipped for genotyping at p14.

\section{2 | TA cloning and DNA sequencing}

PCR primer sequences used in direct sequencing were as follows: exon 2 site: forward: tcctagATTGGTCGCCTGGT; reverse: CCTGGATTATGGCTGGCTGT: exon 3 site: forward: GATTGCTGAGTGCTGGGTTG; reverse: GGGCA GGGACAGTAACATGC.

PCR products from genomic DNA were cloned into pSC-A (Stratagene, Cedar Creek, TX). Plasmid DNA was
(A)

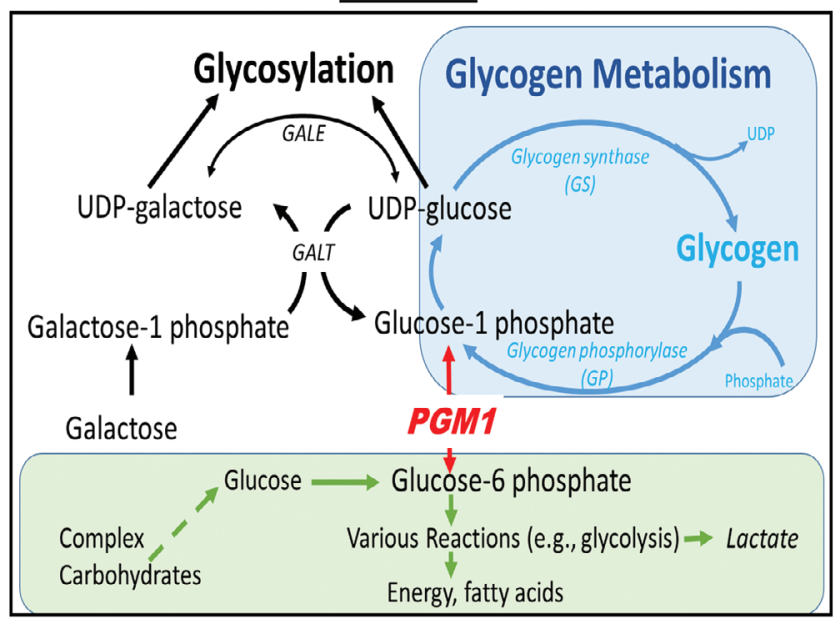

(B)

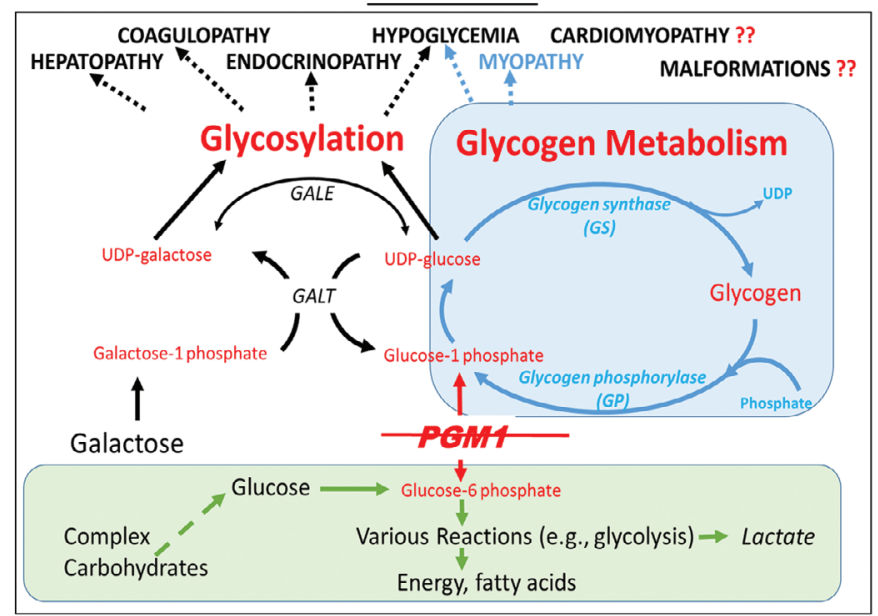

F I G U RE 1 Multi-system involvement in human PGM1-CDG. (A) PGM1 catalyzes the interconversion of glucose-1 phosphate and glucose-6 phosphate and therefore, it plays a regulatory role in glycolysis, glycogenesis, glycogenolysis, and glycosylation. (B) PGM1 deficiency (PGM1-CDG) leads to fasting hypoglycemia, but under normal feeding conditions, there is significant decrease in UDP-glucose and UDP-galactose levels, as well as glucose-1 phosphate and galactose-1 phosphate. As a result, both glycosylation and glycogen metabolism are perturbed, leading to multi-system dysfunctions. CDG, congenital disorder of glycosylation; GALE, UDP-galactose-4' epimerase; GALT, galactose-1 phosphate uridylyltransferase; PGM1, phosphoglucomutase 1 
prepared and sequenced using the BigDye Terminator v3.1 Cycle Sequencing Kit (Applied Biosystems) with M13 forward or reverse primers.

\section{3 | PCR-based genotyping and restriction fragment length polymorphism}

Germline transmission of mutant alleles in subsequent generations was confirmed by a rapid PCR-based genotyping protocol and restriction fragment length polymorphism.

The sequences for the PCR primers used are:

Forward: TGCCTTACAGCACCTCAGTG

Reverse: AACCCAGCACTCAGCAATCC

The PCR product is digested with $B c e$ AI (New England Biolabs cat. no. R0623L) and resolved by a $2 \%$ agarose gel.

\section{4 | Ultrasound studies}

Immediately after mating began, six female mice were monitored by ultrasound daily. Images were acquired with Bmode, and color Doppler modes using a Vevo 2100 high frequency ultrasound machine (VisualSonics) and a MS700 $40 \mathrm{MHz}$ probe. Scan time was approximately 45 minutes to identify number and location of all embryos and obtain images. The timing of the pregnancies was determined based on the developmental stages revealed by the images. As soon as deaths were noted, the pregnant mouse was euthanized and embryos dissected for genotyping.

\section{5 | Timed matings}

Heterozygous $\mathrm{Pgm}^{+/-}$female mice $(\mathrm{n}=6)$ were superovulated with 5 IU of pregnant mare serum and 48-50 hours later with $5 \mathrm{IU}$ of human chorionic gonadotropin. Immediately following injections, female mice were mated to heterozygous male mice. Embryos were harvested at different time points. Genomic DNA was isolated from fetal membranes or whole embryos from E4.5, E9.5, and E15.5 and genotyping was performed by PCR based method as described above.

\subsection{Pgm2 activity assay}

Pgm2 enzyme activity in fibroblasts and tail clips were measured as described previously by Tegtmeyer and coworkers. ${ }^{9}$

\section{7 | Western blot analysis}

The tissue samples were collected from age and sex matched wild type and $\mathrm{Pgm}^{+/-}$mice and the expression of Pgm2 protein was analyzed by Western blot analysis. Briefly, the frozen tissues were homogenized in ice-cold hypotonic lysis buffer (1 mM EDTA, $10 \mathrm{mM}$ Tris-HCl, $\mathrm{pH}$ 7.4), supplemented with protease inhibitor cocktail $(1 \mathrm{mg} / \mathrm{mL}$ each of aprotinin, pepstatin, and leupeptin; $100 \mathrm{mg} / \mathrm{mL}$ phenylmethyl sulfonyl fluoride and $2 \mathrm{mM}$ sodium orthovanadate). Homogenized samples were centrifuged at $18000 \mathrm{~g}$ at $4^{\circ} \mathrm{C}$ for 20 minutes and the supernatant was collected. Protein concentrations were determined by a BCA Protein Assay Kit (Thermo Scientific, Product \# 23223) using a microplate reader. About $40 \mu \mathrm{g}$ of the total protein was resolved by $12 \%$ SDS-PAGE before being transferred to a nitrocellulose membrane. Rabbit anti-human PGM1 antibody (Sigma Aldrich, St. Louis, MO, cat. no. HPA024190) was used as the primary antibody to detect the mouse Pgm2 protein. Primary antibody was detected with IR dyeconjugated secondary antibodies and visualized by Odyssey Image Analyzer (Li-Cor Biotechnology, Lincoln, NE). The abundance of Pgm2 protein in each sample was normalized to the corresponding Gapdh (Sigma Aldrich, St. Louis, MO, cat. no. G9545) abundance detected from the same blot.

\subsection{Galactose rescue experiments in animals}

Galactose rescue experiments were performed by oral supplementation of sterile filtered galactose $(9 \mathrm{mg} / \mathrm{mL}$ in drinking water) to the adult females 1 week before mating and during the course of pregnancies.

\section{9 | Glycome analysis}

Glycome analysis of intact transferrin in serum and fibroblasts was conducted using MALDI-TOF mass spectrometry as previously described. ${ }^{16}$ For transferrin analysis, blood was collected from three age- and sex-matched wild type and $\mathrm{Pgm2}^{+/-}$animals, respectively and sera were collected after clotting. For fibroblast analysis, skin fibroblasts isolated from two age-, sex-matched wild type and $\mathrm{Pgm}^{+/-}$ animals were used to establish primary cell strains, which were used for subsequent analysis.

\section{3 | RESULTS}

\section{1 | Construction of the new Pgm2-KO mouse model}

Similar to humans, mice have multiple Pgm isoforms (Pgm1, Pgm2, and Pgm3) with distinct substrate specificities. ${ }^{14}$ The murine Pgm2 gene encodes an enzyme that belongs to the phosphohexose mutase family and catalyzes the bidirectional interconversion of glucose1-phosphate (Glc-1P) and glucose-6-phosphate (Glc-6P). The mouse Pgm1, on the other hand, catalyzes the conversion of the nucleoside breakdown of ribose-1-phosphate 
(A)

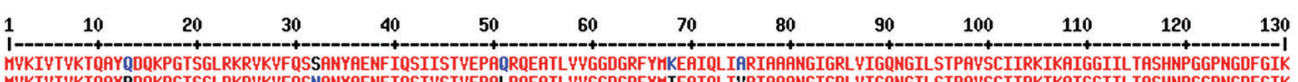

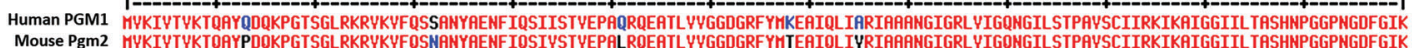
Consensus MYKIVTYKTQAYqDQKPGTSGLRKRYKYFQSnANYAENFIQSI ! STYEPAqRQEATLYYGGDGRFYIKEAIQLIaRTARANGIGRLYIGQNGILSTPAYSCIIRKIKAIGGIILTASHNPGGPNGDFGIK

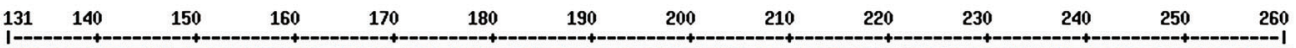

FNISNGGPAPEAITDKIFQISKTIEEYAYCPDLKYOLGYLGKQQFDLENKFKPFTYEIYDSYEAYATMLRSIFDFSHLKELLSGPNRLKICIDAHHGWGPYYKKILCEELGAPANSAYHCYPLEDFGGH

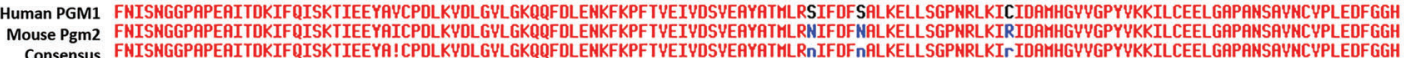

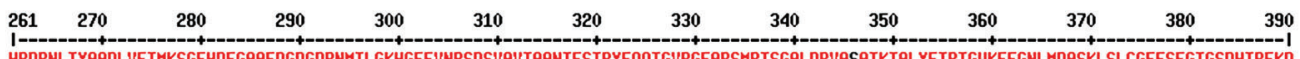
Human PGM1 HPDPNLTYAADLYETHKSGEHDFGAFFDGDGDRNMILGKHGFFYNPSDSVAVIAANIFSIPYFQQTGYRGFARSHPTSGALDRYASATKIALYETPTGHKFFGNLMDASKLSLCGEESFGTGSDHIREKD Consensus HPDPNLTYAADLYETMIKSGEHDFGAFFDGDGDRNMILGKHGFFYMPSDSYAYIAANIFSIPYFQQTGYRGFARSHPTSGRLDRYAnATKIALYETPTGHKFFGNLHDASKLSLCGEESFGTGSDHIREKD

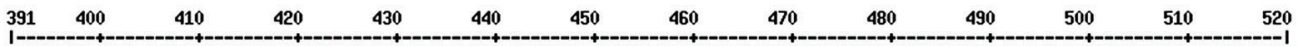

Human PGM1 GLHAYLALLSTLATRKQSYEDTLKDHHQKHGRNFFTRYDYEEYEAEGANKMIIKDLEALMFDRSFYGKQFSANDKYYTYEKADNFEYSDPYDGSISRNQGLRLIFTDGSRTYFRLSGTGSAGATIRLYTDS

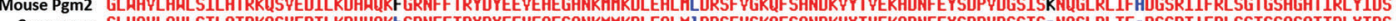

$$
521 \quad 530 \quad 540 \quad 550 \quad 56662
$$

HUMan PGM1 YEKDYAKINQDPQYMLAPLISIALKYSQLQERTGRTAPTYII

Mouse Pgm2 YEKDYAKINQDPQYMLAPLISTALKYSQLQERTGRTAPTYIT

Consensus YEKDYAKINQDPQYMLAPLISTALKYSQLQQERTGRTAPTYIT

(B)

Exon2 Exon3

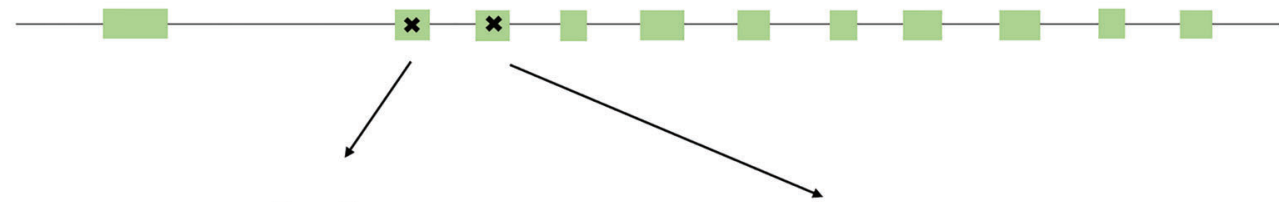

Exon2

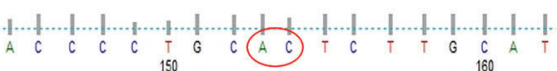

Mutant

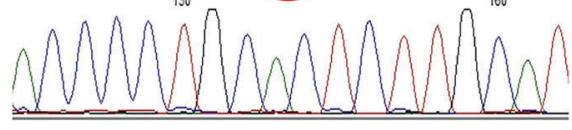

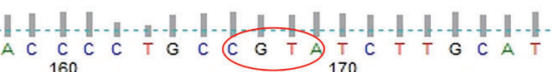

Wild Type

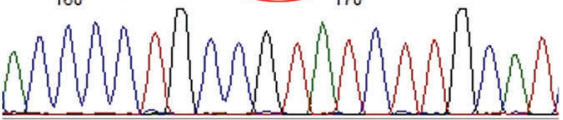

Exon3

, TT T C C C T G A A G G G A A G

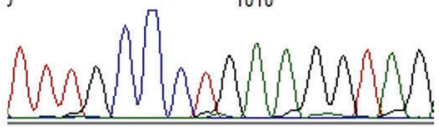

$T_{T} T_{G} C_{1000} \|_{C}$

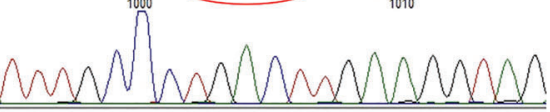

$$
\begin{aligned}
& 5^{\prime} \ldots .(\mathrm{N})_{14} \mathrm{GCCGT} . . .3^{\prime} \\
& 3^{\prime} \ldots \ldots .\left(\mathrm{N}_{112} \mathrm{CGGCA}_{. . .5^{\prime}}\right. \text {... BceAl }
\end{aligned}
$$

(C)

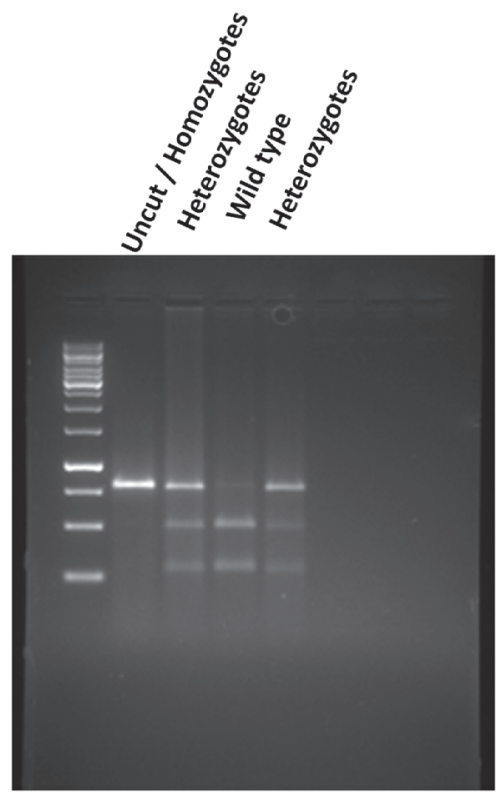

FIGURE 2 Legend on next page. 
or deoxyribose-1-phosphate to the corresponding 5-phosphopentose. Similar to human PGM3, the mouse Pgm3 facilitates the conversion of $N$-acetyl-glucosamine6-phosphate (GlcNAc-6-P) to $N$-acetyl-d-glucosamine1-phosphate (GlcNAc-1-P). ${ }^{17-20}$ Based upon published substrate activities, the mouse Pgm2 enzyme is the equivalent of the human PGM1. To further substantiate this claim, we compared the amino acid sequences encoded by different mouse Pgm genes to that of human PGM1. Figure 2A shows that out of 562 amino acids, the mouse Pgm2 protein shares an identity of $97.7 \%$ (549 a.a. identical). At the cDNA level, Pgm2 cDNA (NM_028132) is 90.11\% identical to its human counterpart (data not shown). Since exons 2 and 3 encode residues that make up the active site and substrate binding site, respectively, ${ }^{21}$ we designed CRISPR reagents that target both exons.

Two pups (one male and one female) born to a pseudopregnant female mouse were tested positive for the disruption of exons 2 and 3 by high resolution melting analysis (data not shown). These mice were crossed to wild-type animals to establish founder animals, which were later crossed to wild type mice to confirm germline transmission. DNA sequencing revealed an insertion/deletion mutation (c. [293_298insAC;293delCGTA]) and a deletion mutation (c.482delTGACT) in exons 2 and 3, respectively (Figure 2B). The CRISPR reagent that targets exon 2 introduced an insertion-deletion frameshift mutation at the GCC codon encoding amino acid alanine 98 (Ala-98) (Figure 2B), which led to the creation of a premature stop codon (TAA) 60 nucleotides downstream, and eliminated the serine -117 at the active site $^{1}$ in the process. The CRISPR reagent that targets exon 3 led to a frameshift and the creation of a premature stop codon (TGA) 38 nucleotides downstream.

\section{2 | Molecular genotyping of Pgm2-KO allele}

The insertion-deletion mutation introduced in exon 2 ablates a BceAI restriction site. Using PCR primers described in "Materials and Methods" section, we were able to amplify a PCR product of $788 \mathrm{bp}$ from the wild-type allele. BceAI restriction digestion resulted in two fragments (504 and $284 \mathrm{bp}$ ) and was used to distinguish between the mutant and wild type alleles (Figure 2C).
TA B L E 1 Embryonic lethality for homozygous $P g m 2^{-/-}$mutants

\begin{tabular}{|lll|}
$\begin{array}{c}\text { Galactose supplementation } \\
\text { in drinking water }\end{array}$ & No & Yes \\
\hline $\begin{array}{c}\text { Number of crosses between } \\
\text { het. parents }\end{array}$ & 15 & 4 \\
\hline $\begin{array}{c}\text { Total number of livebirths } \\
\text { Wild type }\end{array}$ & 78 & 23 \\
Heterozygotes & 23 & 6 \\
Homozygotes & 55 & 17 \\
\hline Chi square value & 0 & 0 \\
\hline$P$ value & 26.692 & 8.39 \\
\hline
\end{tabular}

\section{3 | Embryonic lethality in homozygotes}

Hoping to obtain homozygotes for further studies, we set up crosses between heterozygous parents. After analyzing more than 70 pups from over 10 crosses, we were unable to identify $P g m 2^{-/-}$homozygotes $(P=1.59897 \mathrm{E}-06)$ (Table 1$)$. This suggested embryonic lethality. Moreover, we found four of the heterozygous pups, but none of the wild type, died within 2 days after birth.

To assess the approximate time at which Pgm2deficient embryos succumb in utero, we conducted ultrasound studies on pregnant heterozygous female mice mated with heterozygous males throughout the gestation period. We monitored six pregnancies and our results indicated almost all pregnancies had nine or more living embryos to start with, but two or three embryos from each litter began to show arrested development at around E9.5 (Figure 3). Molecular genotyping of the dissected embryos from the euthanized dams confirmed that embryos succumbed were homozygotes (see Figure 2C). Three of the dead embryos were found to be heterozygotes, but none were wild-type. We also conducted timed mating experiments to reinforce the results of the ultrasound studies. In this case, we followed up to six separate pregnancies. Two pregnant dams were euthanized and dissected at day E4.5. Two were euthanized and dissected at E9.5 and the other two were euthanized and dissected at E15.5. Similar to what we found in the ultrasound work, 8 to 10 embryos were seen in all pregnancies as revealed from pregnant dams euthanized at E4.5 (Figure S1), but

F I G URE 2 Construction of traditional Pgm2-KO mice. (A) Amino acid sequences of mouse Pgm2 to that of human PGM1 were compared using MultAlin software (http://multalin.toulouse.inra.fr/multalin/). Out of 562 amino acids, the mouse Pgm 2 protein shares an identity of $97.7 \%$ (549 a.a. identical). (B) Two CRISPR reagents were used to introduce an insertion-deletion and a deletion, respectively in exons 2 and 3 of the mouse Pgm2 gene. The frameshift mutation introduced in exon 2 created a premature stop codon and eliminated a BceAI restriction site that was used in PCR-based genotyping. (C) Genomic DNA isolated from the mice are amplified by PCR method and digested with restriction endonuclease BceA1, producing $788 \mathrm{bp}$ from the uncut or homozygote (lane 2), 788, 504, and $284 \mathrm{bp}$ from heterozygote (lanes 3 and 5 ) and 504 and 284 bp for the wild-type allele (lane 4). KO, knockout 


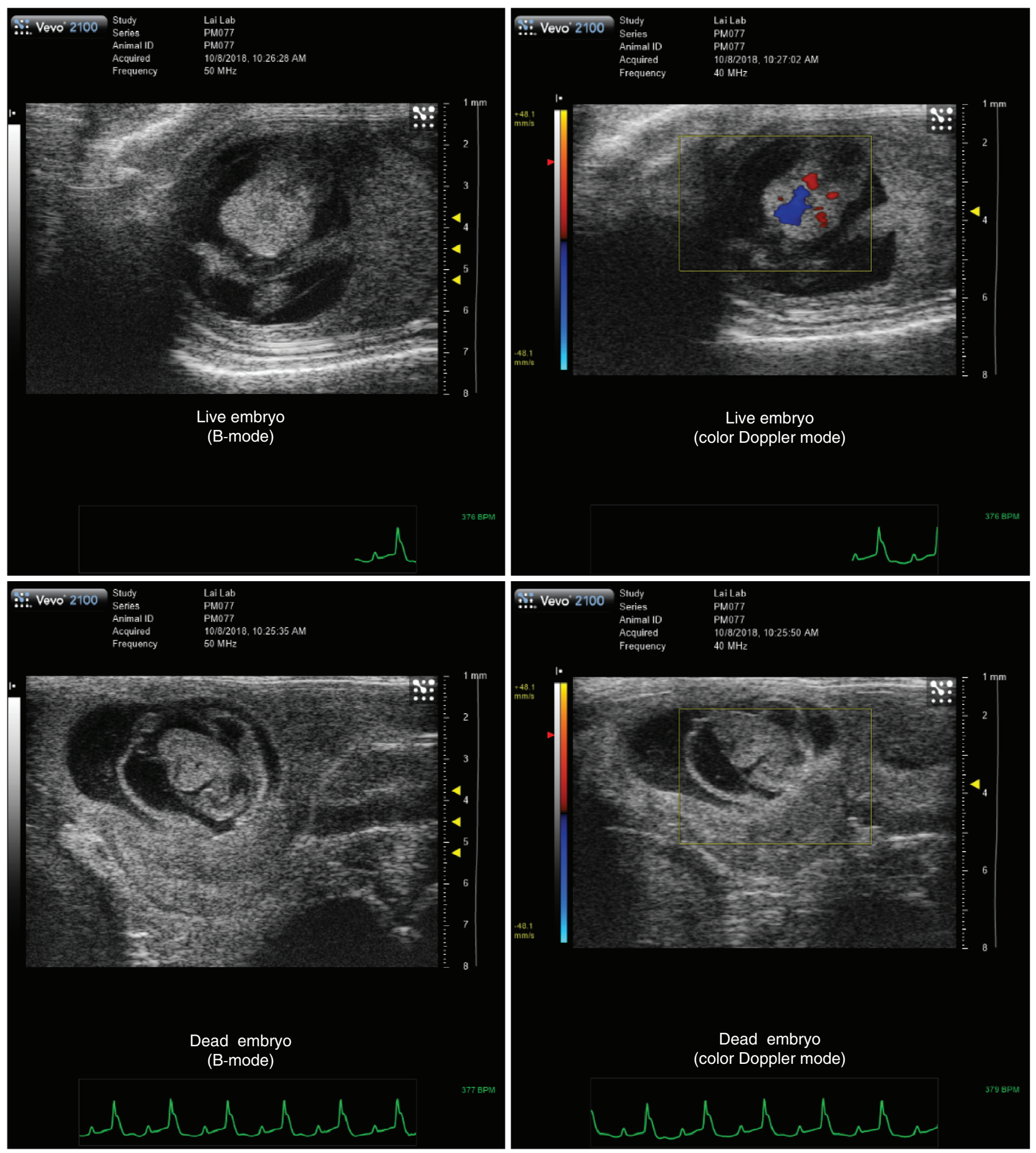

F IG URE 3 Use of ultrasound imaging to follow the course of pregnancies in $P g m 2^{+/-}$females mated to $P g m 2^{+/-}$males. Six pregnancies were followed by ultrasound daily soon after matings began. Color Doppler mode reveals blood flow in the live fetus (upper right panel). The representative figures showed that no blood flow was detected in an E9.5 embryo

some of the embryos became arrested shortly before E9.5 (Figure S1). Molecular genotyping confirmed the dead embryos in six pregnancies were homozygotes, as well as four heterozygotes.
We also attempted to rescue the lethality phenotype by oral galactose supplementation based upon the dosage used in human patients, ${ }^{9,13}$ but we observed no effects at the dosing regimen we employed (Table 1). 


\section{4 | Biochemical studies of heterozygous $\mathbf{P g m}^{+/-}$animals and cells}

Since we could not obtain any homozygous $P g m 2^{-1-}$ livebirths, we confirmed the knockout of the Pgm2 gene in the heterozygous animals by measuring the Pgm 2 activity in their skin fibroblasts and tissues (tail clips). Figure 4A,B shows significant (more than 60\%) reduction of Pgm2 activity in the fibroblasts and tail clips in the heterozygous animals, which was reflected in the Western blot analysis of the corresponding tail clip samples (Figure 4C).

\section{5 | Glycome analysis}

Glycoprofiling in PGM1 deficiency in humans shows a recognizable pattern with changes in three glycan indexes: (a) normal glycosylation, (b) lack of complete glycans, and (c) lack of galactose residues, with decrease in total galactosylation and sialylation. There is also frequently an increase in high mannose glycans and fucosylation of total plasma glycans. ${ }^{22}$

Our glycomics studies in heterozygous $\mathrm{Pgm}^{+/-}$mice showed a distinct glycosylation pattern compared to the glycosylation pattern in wild type mice, but different from the human PGM1 deficient pattern. In the serum analysis of intact transferrin in heterozygous $\mathrm{Pgm}^{+/-}$mice there was a profound decrease of the tetrasialotransferrin glycoform (type I), and a relative increase of truncated glycans (type II pattern) (Figure 5). (Please note that mice serum has a relative increase in truncated glycans in wild type mice, compared to healthy human serum.) The MALDI-TOF analysis of total glycans of in heterozygous $\mathrm{Pgm}^{+/-}$mice serum showed no abnormalities. There was some difference in the global galactosylation pattern with increased presence of total ungalactosylated truncated glycans in heterozygous $\mathrm{Pgm}^{+/-}$mice, and there was no terminal sialylation observed in the heterozygous fibroblasts, similar to the pattern seen in human fibroblasts. However, there was no increase in mannosylation and fucosylation. No apparent loss-of-glycans were detected. These changes suggest a glycan-processing defect in heterozygotes, but different from biallelic PGM1 mutant cells in humans.

\section{4 | DISCUSSION}

To understand the pathophysiology of PGM1 deficiency and to facilitate the development of novel therapeutics, we here report the construction of a constitutive Pgm2-KO mouse model using CRISPR-Cas9 technology (Figure 2). By comparing the amino acid sequences of different isoforms to the human PGM1, we confirmed that the mouse Pgm2 protein shares the highest identity with human PGMI (Figure 2A) and therefore, we chose to target the Pgm2 gene in this
(A)

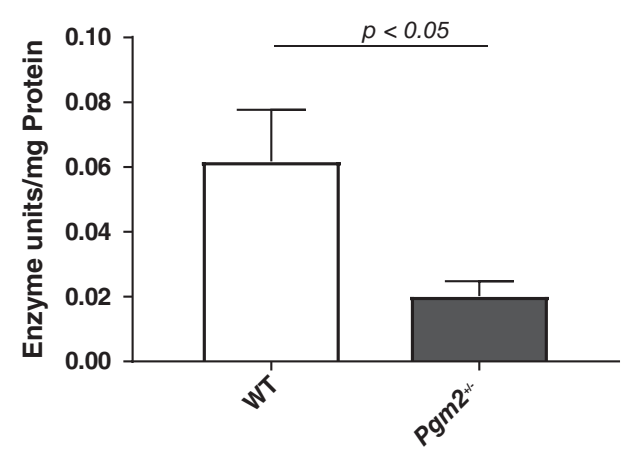

(B)

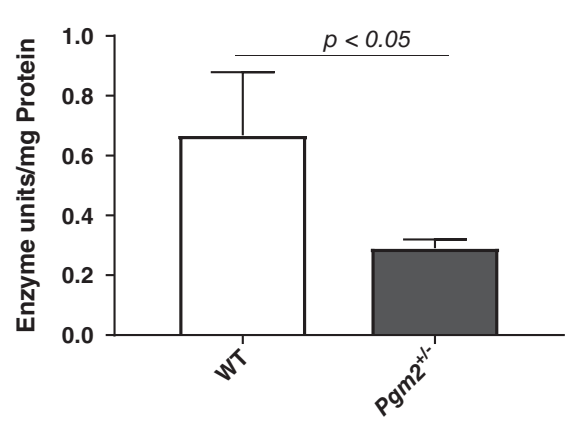

tail clips

(C)

(C) $\quad$ - tail clips

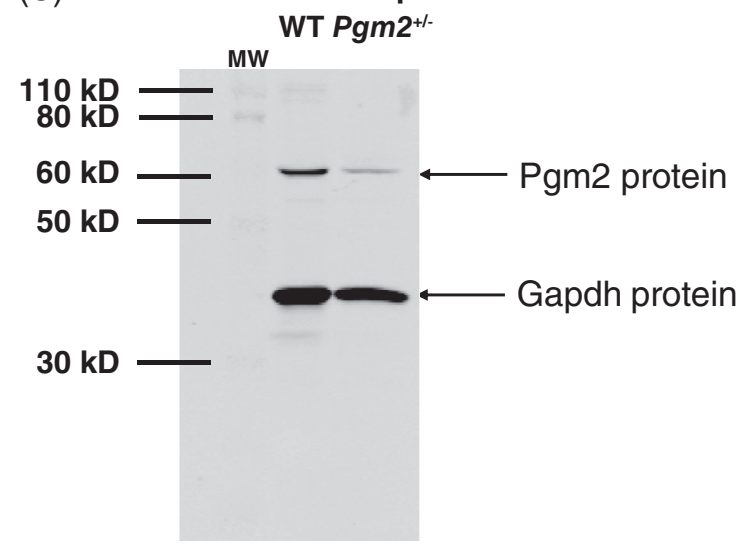



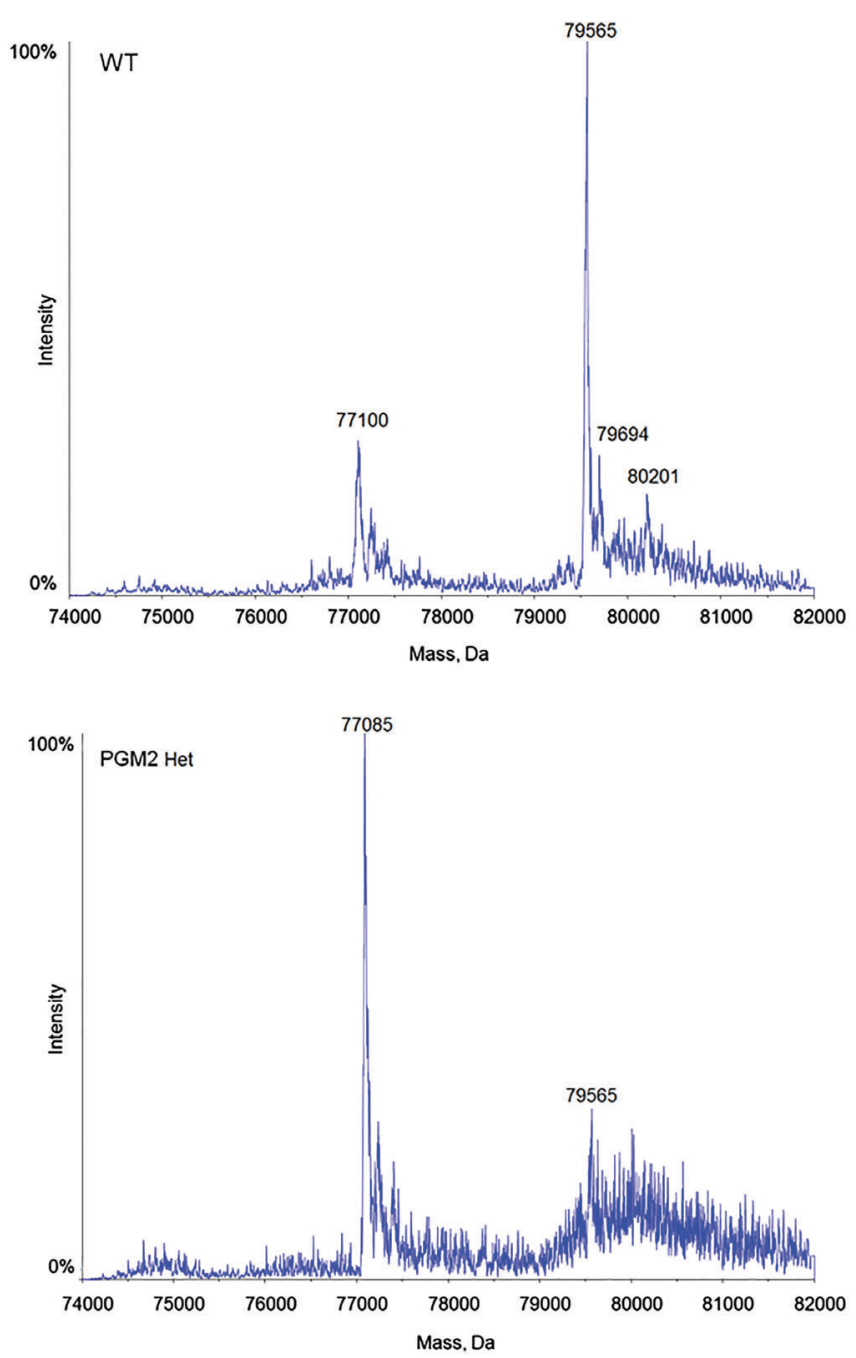

F I G U RE 5 Abnormal glycosylation of serum transferrin in $\mathrm{Pgm2}^{+/-}$heterozygotes. Representative transferrin spectra in wild type and heterozygous $\mathrm{Pgm2}^{+/-}$mice serum evaluated by mass spectrometry. In the heterozygous mice di-oligo (tetrasialo) transferrin was absent and there was a significant increase in the abundance of truncated glycans

study. The insertion/deletion at exon 2 and the deletion mutation in exon 3 each create a premature stop codon in the respective exon by itself. In addition, both mutations led to frameshifts alone or in combination. Therefore, the chance for a functional Pgm2 protein to be produced through single/double read-throughs is virtually nonexistent.

In this study, we demonstrated that homozygosity for Pgm2-KO mutations leads to embryonic lethality in mice (Table 1, Figures 3 and S1). While other mouse models of CDG, such as Pmm2-KO mice, also exhibited lethality for the homozygous embryos, our Pgm2-deficient model presents some differences. ${ }^{23,24}$ As to the Pmm2-KO mouse, no Pmm2-null embryos were recovered beyond the embryonic day 3.5. ${ }^{24}$ Yet, our Pgm2-deficient embryos appeared to perish shortly before E9.5 (Figures 3 and S1). Could this be due to the existence of multiple Pgm isoforms which partially compensate for the loss of Pgm2 activity and sustain embryonic development till mid-gestation when organogenesis begins when the demand for Pgm2 function is so critical that even the other isoforms are insufficient? Regardless, we can conclude that no protein in the mouse that compensates for Pgm2 for survival. At the moment, it is unclear why the KO animals perish in utero. Tamplin and coworkers showed that $\mathrm{Pgm} 2$ is expressed abundantly in the heart around E8.5. ${ }^{25}$

Surprisingly, some $\mathrm{Pgm2}^{+/-}$embryos did not come to full term either, while we did not see any deaths of wild type embryos throughout our studies. In addition, 7\% of heterozygous newborns died soon after birth. This suggests that $\mathrm{Pgm}^{+/-}$mice are seemingly more affected than heterozygous patients, but it should be noted that there are no formal retrospective or longitudinal studies of the human carriers. Remarkably, when we assessed the Pgm2 enzymatic activity in the tissues or cultured skin fibroblasts from the surviving $\mathrm{Pgm2}^{+/-}$animals, we revealed more than $60 \%$ reduction in enzymatic activity, with a few in the $30 \%$ to $40 \%$ range. Could the unusually low Pgm2 activity in the heterozygous animals, which potentially can be treated like human symptomatic patients who harbor hypomorphic mutations, explain their worse-than-expected phenotypes? While it is beyond the scope of the current work to investigate the mechanisms that account for the lower than expected Pgm2 activity in the heterozygotes, but we will examine any potential dominant negative effect of the heterozygous KO mutation, as well as other potential epigenetic mechanisms in the future.

The pathological condition in the heterozygous animals was also reflected in the abnormal glycosylation studies of the serum transferrin and skin fibroblasts harvested from heterozygous animals. In both cases, mass spectrometry showed increased levels of truncated glycans with decreased galactosylation (Figures 5 and S2), which is one of the characteristic features in human PGM1-CDG patients carrying biallelic mutations. ${ }^{13,26}$ Therefore, our heterozygous mouse model mirrors an important aspect of the human phenotype. These heterozygous mice also showed loss of serum glycans, which is the other diagnostic feature of PGM1-deficient patients. Therefore, our findings suggest that a significant reduction of Pgm2 activity in the carrier cells is sufficient to cause type I glycosylation defects in the cytosol and the ER.

Schneider and colleagues reported that mannose supplementation rescued the embryonic lethality phenotype in the Pmm2 ${ }^{R 137 H / F 118 L}$ model, ${ }^{27}$ but Chan and coworkers were unsuccessful in their attempt with the same amount of mannose in Pmm2 $2^{R 137 H / F 115 L}$ model, which showed 62\% embryonic lethality in homozygotes. However, they were able to significantly rescue embryonic lethality in the

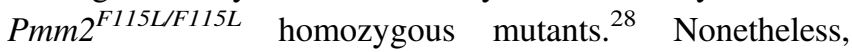


Wong and colleagues were able to reverse some of the biochemical phenotypes of PGM1 deficiency in human patients and cells with hypomorphic mutations, despite the fact that not all phenotypes were corrected. ${ }^{11,13}$ Therefore, we examined effects of galactose supplementation at $9 \mathrm{mg} / \mathrm{mL}$ (drinking water) based upon the dosage used in human patients $(1.5 \mathrm{~g} / \mathrm{kg} / \mathrm{day})$, but were unable to correct any mouse phenotype, in particular the reversal of embryonic lethal phenotype of the homozygotes (Table 1). In hindsight, our hope for rescue might be over-ambitious because after all, ours is a complete $\mathrm{KO}$ mouse model vs a hypomorphic human one. We can conclude that even under galactose treatment, no other enzyme can take over the role of Pgm2. In the future, we will evaluate the effects of galactose supplementation on other biochemical markers in the heterozygous (hypomorphic) mutants.

\section{ACKNOWLEDGMENTS}

Research support include the Primary Children's Hospital Foundation K2R2R Award (to K.L.), UDN Metabolomics Core and FP00096621 (National Center for Advancing Translational Sciences) grants (to E.M.), and NIH S10 RR027506-01 grant awarded to K.J.W. at the University of Utah Small Animal Ultrasound Core.

\section{CONFLICT OF INTEREST}

The authors declare no potential conflict of interest.

\section{AUTHOR CONTRIBUTIONS}

B.B. and K.L. contributed in planning and performance of the experiments, interpretation of the results, and composition of the manuscript. J.V. performed the experiments and interpreted the results. A.L., C.T., Y.Y., and K.L.C. performed the experiments. K.R. reviewed experimental data. K.W. contributed in planning of the experiments. T.K and E.M. contributed in planning of the experiments, interpretation of the results, and composition of the manuscript.

\section{ETHICS STATEMENT}

This research does not involve human subjects and therefore, IRB approval is not required. This research is conducted in strict adherence to protocol approved by the ICAUC of University of Utah.

\section{ORCID}

Kent Lai (iD https://orcid.org/0000-0002-2377-9905

\section{REFERENCES}

1. Beamer L. Mutations in hereditary phosphoglucomutase 1 deficiency map to key regions of enzyme structure and function. J Inherit Metab Dis. 2015;38:243-256.

2. Ondruskova N, Honzik T, Vondrackova A, Tesarova M, Zeman J, Hansikova H. Glycogen storage disease-like phenotype with central nervous system involvement in a PGM1-CDG patient. Neuroendocrinol Lett. 2014;35:137-141.

3. Voermans NC, Preisler N, Madsen KL, et al. PGM1 deficiency: substrate use during exercise and effect of treatment with galactose. Neuromuscul Disord. 2017;27:370-376.

4. Jaeken J. Congenital disorders of glycosylation (CDG): it's (nearly) all in it! J Inherit Metab Dis. 2011;34:853-858.

5. Kucukcongar A, Tumer L, Ezgu FS, et al. A case with rare type of congenital disorder of glycosylation: Pgm1-Cdg. Genet Couns. 2015;26:87-90.

6. Perez B, Medrano C, Ecay MJ, et al. A novel congenital disorder of glycosylation type without central nervous system involvement caused by mutations in the phosphoglucomutase 1 gene. $J$ Inherit Metab Dis. 2013;36:535-542.

7. Loewenthal N, Haim A, Parvari R, Hershkovitz E. Phosphoglucomutase-1 deficiency: intrafamilial clinical variability and common secondary adrenal insufficiency. Am J Med Genet A. 2015;167: 3139-3143.

8. Stojkovic T, Vissing J, Petit F, et al. Muscle glycogenosis due to phosphoglucomutase 1 deficiency. $N$ Engl J Med. 2009;361: 425-427.

9. Tegtmeyer LC, Rust S, van Scherpenzeel M, et al. Multiple phenotypes in phosphoglucomutase 1 deficiency. N Engl J Med. 2014; 370:533-542.

10. Timal S, Hoischen A, Lehle L, et al. Gene identification in the congenital disorders of glycosylation type I by whole-exome sequencing. Hum Mol Genet. 2012;21:4151-4161.

11. Morava E. Galactose supplementation in phosphoglucomutase-1 deficiency; review and outlook for a novel treatable CDG. Mol Genet Metab. 2014;112:275-279.

12. Radenkovic S, Bird MJ, Emmerzaal TL, et al. The metabolic map into the pathomechanism and treatment of PGM1-CDG. Am J Hum Genet. 2019;104:835-846.

13. Wong SYW, Gadomski T, van Scherpenzeel M, et al. Oral Dgalactose supplementation in PGM1-CDG. Genet Med. 2017;19: 1226-1235.

14. Shows TB, Ruddle FH, Roderick TH. Phosphoglucomutase electrophoretic variants in mouse. Biochem Genet. 1969;3: 25-35.

15. Lin S, Staahl B, Alla RK, Doudna JA. Enhanced homologydirected human genome engineering by controlled timing of CRISPR/Cas9 delivery. Elife. 2014;3:e04766.

16. Ferreira CR, Xia ZJ, Clement A, et al. A recurrent de novo heterozygous COG4 substitution leads to Saul-Wilson syndrome, disrupted vesicular trafficking, and altered proteoglycan glycosylation. Am J Hum Genet. 2018;103:553-567.

17. Greig KT, Antonchuk J, Metcalf D, et al. Agm1/Pgm-3-mediated sugar nucleotide synthesis is essential for hematopoiesis and development. Mol Cell Biol. 2007;27:5849-5859.

18. Konno R, Niwa A, Yasumura Y. Linkage of the Dao-1 gene for D-amino-acid oxidase to the Pgm-1 gene for phosphoglucomutase-1 on the mouse chromosome-5. Jpn J Genet. 1989;64:341-345. 
19. Muenks AG, Stiers KM, Beamer LJ. Sequence-structure relationships, expression profiles, and disease-associated mutations in the paralogs of phosphoglucomutase 1. PLoS One. 2017;12: e0183563.

20. Stray-Pedersen A, Backe PH, Sorte HS, et al. PGM3 mutations cause a congenital disorder of glycosylation with severe immunodeficiency and skeletal dysplasia. Am J Hum Genet. 2014;95: 96-107.

21. Stiers KM, Kain BN, Graham AC, Beamer LJ. Induced structural disorder as a molecular mechanism for enzyme dysfunction in phosphoglucomutase 1 deficiency. J Mol Biol. 2016;428:14931505.

22. Abu Bakar N, Voermans NC, Marquardt T, et al. Intact transferrin and total plasma glycoprofiling for diagnosis and therapy monitoring in phosphoglucomutase-I deficiency. Transl Res. 2018;199: 62-76.

23. DeRossi C, Bode L, Eklund EA, et al. Ablation of mouse phosphomannose isomerase (Mpi) causes mannose 6-phosphate accumulation, toxicity, and embryonic lethality. J Biol Chem. 2006; 281:5916-5927.

24. Thiel C, Lubke T, Matthijs G, von Figura K, Korner C. Targeted disruption of the mouse phosphomannomutase 2 gene causes early embryonic lethality. Mol Cell Biol. 2006;26:5615-5620.

25. Tamplin OJ, Kinzel D, Cox BJ, Bell CE, Rossant J, Lickert H. Microarray analysis of Foxa2 mutant mouse embryos reveals novel gene expression and inductive roles for the gastrula organizer and its derivatives. BMC Genomics. 2008;9:511.

26. Witters $\mathrm{P}$, Cassiman D, Morava E. Nutritional therapies in congenital disorders of glycosylation (CDG). Nutrients. 2017;9:1222.

27. Schneider A, Theil C, Rindermann J, et al. Successful prenatal mannose treatment for congenital disorder of glycosylation-Ia in mice. Nat Med. 2011;18:71-83.

28. Chan B, Clasquin M, Smolen GA, et al. A mouse model of a human congenital disorder of glycosylation caused by loss of PMM2. Hum Mol Genet. 2016;25:2182-2193.

\section{SUPPORTING INFORMATION}

Additional supporting information may be found online in the Supporting Information section at the end of this article.

How to cite this article: Balakrishnan $\mathrm{B}$, Verheijen J, Lupo A, et al. A novel phosphoglucomutase-deficient mouse model reveals aberrant glycosylation and early embryonic lethality. J Inherit Metab Dis. 2019;42:998-1007. https://doi. org/10.1002/jimd. 12110 